\title{
Une façon d'indiquer la « non-coïncidence entre les mots et les choses $"$, on va dire...
}

\author{
Emmanuelle Labeau \\ Aston University \\ e.labeau@aston.ac.uk
}

\section{Introduction}

La grammaticalisation de la séquence aller + infinitif en marqueur de temporalité est suffisamment avancée pour figurer jusque dans les méthodes d'enseignement du français langue étrangère. En Angleterre, les examinateurs du A-level (baccalauréat) attribuent des points de bonus pour l'utilisation à l'oral du futur périphrastique, censé signifier une maîtrise de la langue quotidienne. Toutefois, la séquence aller + infinitif est loin de se limiter à l'expression de l'ultériorité comme Bres et Labeau l'ont montré dans plusieurs articles (Bres et Labeau 2012; Bres et Labeau, à par. entre autres). Malgré la diversité des effets de sens en discours, Bres et Labeau postulent que ceux-ci peuvent tous s'expliquer par l'interaction avec différents éléments contextuels de la valeur originale de mouvement. La présente étude s'inscrira dans la même lignée.

Nous souhaitons nous pencher ici sur un emploi particulier qui n'a fait l'objet - à notre connaissance - que d'un article (Lansari 2010). Cet emploi «modalisant» que Lansari limite à la formule on va dire mérite d'être approfondi pour plusieurs raisons. D'une part, l'emploi n'est décrit que sur la base de «vingt exemples tirés d'internet, de blogs ou de forums » (Lansari 2010 : 120) alors que, de l'aveu de Lansari elle-même, l'emploi relève de l'oral. Il serait donc utile d'enrichir - quantitativement et qualitativement - le corpus et d'y intégrer des occurrences d'oral authentique. D'autre part, Lansari restreint l'emploi modalisant à la séquence on va dire ; on pourrait s'interroger sur la capacité de séquences comme je vais dire à remplir les mêmes fonctions discursives.

Dans cet article, nous commencerons par un - forcément bref - état de la question. Après avoir présenté le corpus, nous testerons les hypothèses précédemment défendues à la lueur du corpus rassemblé.

\section{Etat de la question}

L'emploi modalisant de «on va dire » est un phénomène peu étudié par les linguistes. Nous nous efforcerons dans cette section de résumer ce qui en a été dit.

Lansari (2010) ne relève de mentions de cet usage que chez Schneider (2007) et base ses propres conclusions sur un corpus limité. L'effet de sens est cependant relevé par les journalistes comme le montrent les citations suivantes empruntées à Lansari $(2010: 120)$ :

«on va dire signifie généralement que 1) on hésite à dire, 2) on pense exactement le contraire de ce qu'on dit, 3) on n'a rien à dire [...]. Dans la plupart des cas de figure, on va dire signifie on vient de dire, puisque l'expression suit l'énoncé de la réponse. On va dire gagnerait, grammaticalement et sémantique, à se dire $j$ 'ai dit » (C. Sorg, chronique « on va dire entre guillemets » Télérama $\mathrm{n}^{\circ} .2814$, décembre 2003). 
«on va dire : expression servant à ne pas assumer totalement la responsabilité de ce qui va suivre. S'exprimait jadis par: Je dirais volontiers si j'osais (H. Viala, article « Des mots 'total' mode, on va dire ». Le Monde, 24 avril 2006).

\subsection{Restrictions d'usage}

D'après Lansari (2010), la séquence on va dire est sujette à une série de restrictions dans les contextes d'emploi, la syntaxe et la position phrastique.

\subsubsection{Contextes d'emploi}

D'abord, il s'agirait d'un emploi majoritairement oral, néanmoins attesté dans un genre écrit peu soutenu comme le blog. Lansari (2010 : 119) n'en a relevé qu'un exemple littéraire dans un roman contemporain, ce qui suggère un emploi récent :

(1) Il n'était pas avec moi, l'encombrant amoureux toutou malodorant. Mais il était là, lui. Mon siamois! Et ses yeux ont brillé, brillé. Et il a fait un.. on va dire que ce fut un feulement. Je sais que ce sont les tigres qui feulent. Cette nuit-là, mon siamois, il est devenu un tigre. Et moi une chatte chavirée. (Frantext, R971, R.Forlani, Gouttière, 1989, p.153, cité par Lansari $2010: 119)$

\subsubsection{Le figement}

Lansari (2010) avance que la séquence on va dire est « éminemment figé[e] », même s'il reste possible d'intercaler des éléments entre l'auxiliaire et l'infinitif (ex. : on va juste dire). D'abord, elle est censée ne se réaliser qu'avec on et est exclue avec je (2010:120), même si ce on est décrit plus loin comme "glosable par je ». De plus, elle se limite apparemment à la forme affirmative (p.133) même si cette affirmation est quelque peu atténuée en effet, Lansari (2010 : 134) donne cet exemple oral :

(2) [le locuteur parle de sa grand-mère, victime d'un accident vasculaire cérébral quelques mois auparavant] Elle s'en est, non on va pas dire complètement sortie, mais ça va (émission télévisée)

Finalement, alors que aller + infinitif dans son emploi ultérieur se réalise avec le présent et l'imparfait, seul le premier tiroir semble possible ici (p.123).

\subsubsection{La position phrastique}

La position phrastique de la séquence ne fait pas l'unanimité. Selon Sorg (2003), on va dire est situé après l'énoncé mais pour Viala (2006), on va dire le précède. Conformément aux critères syntaxiques de la rection faible (Apothéloz 2003) qui caractérise le verbe dire, on va dire est susceptible de fonctionner sans complétive en postposition ou en incise. Lansari identifie trois structures possibles :

(a) on va dire + subordonnée complétive :

(3) [A propos d'une photographie] On va dire poliment que c'est vraiment raté. (Lansari $2012: 121)$ 
Il s'agit du « cas le plus prototypique, et semble-t-il le plus fréquent dans les exemples - écrits, rappelons-le - que nous avons relevés [...] » (p.120). Lansari en donne l'interprétation suivante : «Même s'il n'est pas question de renvoi à l'avenir ou d'ultériorité temporelle, aller + inf. permet d'annoncer un nouvel élément, ou de faire une mise au point nécessaire pour la suite du discours $\gg(\mathrm{p} .121)$.

\section{(b) on va dire + adjectif / GN :}

(4) [sur un blog] ça fait longtemps, je crois que je n'avais pas fait ma crise « je hais les lundis ». Parce que mes nouveaux lundi, c'est : se lever à six heures (et essayer de ne pas avoir trois quarts d'heure de retard comme la semaine dernière), une demi-heure pour manger (en fait, on va dire une dizaine de minutes, le temps de s'en griller une avant et de faire la queue au micro-ondes pour réchauffer la gamelle), [...] (Lansari $2010: 121)$

Interprétation offerte par Lansari : «Ici, la périphrase a surtout pour effet de mettre en valeur l'adjectif ou le groupe nominal choisi » (p.122)

\section{(c) on va dire en position finale :}

«Et c'est, d'après nos observations, cette configuration que l'on retrouve le plus dans du véritable oral, à la radio ou à la télévision. Elle est moins fréquente dans notre corpus d'exemples uniquement écrits, mais elle n'en est pas non plus totalement absente » (p.122).

(5) [recette du bœuf au curry] Ça, c'est la version «officielle» on va dire. J'ai rajouté quelques légumes dont 2 petits oignons nouveaux, 1 oignon [...] (Lansari 2010 122)

L'interprétation que propose Lansari est la suivant : «on va dire apparait en position finale, pour commenter ce qui vient d'être dit » (p.122).

\subsection{Interprétation de on va dire comme modalisateur}

On va dire semble indiquer qu'on n'assume pas la responsabilité de l'énoncé (Viala 2006) ou qu'on le prend avec hésitation (Sorg 2003). Plus conceptuellement, il s'agit pour Lansari (2010) d'une « modalisation autonymique ${ }^{1}$ méta-énonciative ${ }^{2}$ » avec deux types de modalisation :

(a) quantitative (référant à la (non-)existence d'un événement) et donc « liée à la connaissance ou méconnaissance, et peut à ce titre être qualifiée d'épistémique » :

(6) - Quelle a été ta plus grande satisfaction en tant que photographe?

- Dur dur, ben... je pense que sa sera de réussir le mariage de mon ami qui se déroule après demain (le 6 janvier), sinon ben, je ne sais pas. Sa fait toujours plaisir de savoir que certaines photos plaisent. On va dire que je suis satisfait lorsque la photo que j'ai prise est exactement comme l'idée que j'imaginai [...]. Pas facile. (j'ai pas trop saisi la question, désolé). (Lansari $2010: 127)$

(7) [à propos d'une enquête] Moi j'dis, y a un truc qui colle pas quand même !!!! on va juste dire alors qu'il y a plus de personnes de sexe féminin qui cotoie mon blog et que les hommes...sont des extra-terestres!! Remarquez, j'avais pas trop de doutes à ce sujet !!!! noooooon on va dire que ceux qui ont répondu ça sont des gens comme moi, qui aiment délirer ! (Lansari 2010 : 127) 
(b) qualitative ${ }^{3}$ : ce type de modalisation indique la «non-coïncidence entre les mots et les choses » (Authier-Revuz 1995) : voir la marque d'hésitation heu en (8) et les guillemets cidessus en (5) :

(8) [Sur un blog, commentaire illustrant une photo de dallage d'une terrasse] Dallage heu, rustique, on va dire. (Lansari $2010: 128$ )

(9) pr info je croi que quelqu'un demander la ferrite ça sert a eliminé les parasite sur le cable ça lisse la tension en gros, $\mathrm{c}$ une sorte de metal un anneau on va dire et tu enroule le fil dedans ce qui crée un effet bobine plus tu fais de tout plus tu augmente l'effet bobine mais plus tu augmente les risques qd tu débranche l'alim [...] voilà j'espere que je suis pas trop bordelique ds mon explication [...]. (Lansari $2010: 129$ )

(10) J'ai une petite déformation de l'esprit (on va dire un petit excès d'esprit cartésien) qui me fait réagir quand je lis des énoncés imprécis ou infondés. (Lansari $2010: 129)$

«dans ces exemples, l'énonciateur a d'abord choisi un premier élément (une sorte de métal $[\ldots]$, tout le monde [...], déformation de l'esprit [...] puis il a introduit par le biais de on va dire un second élément lui permettant de modifier ou de nuancer ce premier élément. »(p.129) :

(11) [à propos du film Die Hard 4] Allez on va dire que si McClane c'est assagi c'est parce qu'il a prit de la bouteille. Je sais c'est con comme excuse mais sachez juste que le père Willis ne cesse de gueuler ses derniers temps pour tous ses films qui passent à côté de leurs buts à cause de différentes censures, que monsieur n'approuve pas, donc soyons compatissant avec notre Bruce. (Lansari 2010 : 131).

\section{Notre contribution}

La réflexion lancée par Lansari (2010) nous semble remarquable à plus d'un titre. Toutefois, elle nous semble perfectible à au moins trois niveaux. D'abord, alors que la séquence on va dire est supposée appartenir à l'oral, le corpus sur lequel s'est basée Lansari est presqu'exclusivement écrit. Ceci nous semble susceptible de biaiser la réflexion et c'est pourquoi nous enrichirons le corpus de données conversationnelles contemporaines authentiques. Ensuite, le figement présumé de la séquence que défend Lansari est contredit par des exemples de son corpus pourtant limité. L'examen d'un corpus plus étoffé nous permettra sans doute de préciser les contraintes pesant sur la séquence. Enfin, une comparaison avec d'autres formes du paradigme de dire pourrait permettre de mieux cerner la valeur sémantique de la séquence qui nous intéresse.

\subsection{Le corpus}

Compte tenu de l'oralité assumée de la séquence étudiée, nous avons exploré trois corpus oraux

(a) Le corpus CFPP2000 issu du projet Discours sur la ville. Corpus de Français Parlé Parisien des années 2000 (disponible en ligne à http://cfpp2000.univ-paris3.fr/Corpus.html). CFPP2000 donne la parole à 41 informateurs en 28 interviews (2198 min) et a généré 96 occurrences de on va dire modalisant.

(b) Le corpus CLAPI interrogeable en ligne à http://clapi.univlyon2.fr/analyse requete aide.php?menu=outils. Il comprend 45 heures d'interactions et offre 12 exemples de on va dire modalisant. 
(c) Un corpus personnel d'interviews (163 min) réalisées pendant l'année académique 2009-10 auprès de cinq étudiants Erasmus français grâce au soutien d'une bourse de la Délégation Générale à la Langue Française et aux Langues de France (DGLFLF). Les entretiens avec une assistante de recherche, basés sur les thèmes suivants, étaient supposés générer l'emploi d'une variété de temps verbaux :

- $\quad$ Récits de rêve (imparfait)

- $\quad$ Récits biographiques (personnage historique vs autobiographie) (PC vs PS)

- $\quad$ Narration de film vs d'épisode historique (PC/ PRES vs PS)

- $\quad$ Présentation de projets d'avenir vs conjectures (Futur périphrastique ou simple)

Le corpus contient dix-sept occurrences de on va dire générés par deux des cinq informateurs : 15 par A. et 2 par J.

Notre réflexion se basera donc sur 125 occurrences orales de on va dire.

\subsection{Les hypothèses à vérifier}

Dans cette section, nous allons tester sur la base de notre corpus oral plus étendu certaines des hypothèses avancées par Lansari à savoir (i) les trois configurations possibles décrites en 2.1.3 et (ii) le figement supposé de la séquence on va dire. Nous essaierons aussi de tester son hypothèse sur (iii) la récence d'apparition.

\subsubsection{Trois configurations possibles}

Lansari avance que on va dire modalisant peut apparaître dans trois configurations (i) suivi d'une complétive ; (ii) devant un groupe nominal ou un adjectif et (iii) en position finale. Le tableau ci-dessous résume les configurations présentes dans nos corpus oraux.

\begin{tabular}{|l|r|r|r|r|r|}
\hline & CFPP2000 & CLAPI & DGLFLF & total & Total (\%) \\
\hline On va dire (que) & 16 & 4 & 2 & 22 & 22,4 \\
& $\begin{array}{r}\text { (sans } \\
\text { que) }\end{array}$ & $\begin{array}{r}3 \text { (sans } \\
\text { que) }\end{array}$ & - & 6 & \\
\hline On va dire + Adj / GN & 26 & 1 & - & 27 & 21,6 \\
\hline On va dire final & 7 & 3 & 6 & 16 & 12,8 \\
\hline Autres : & & & & & \\
Indication de lieu + on va dire & 4 & - & - & 4 & 3,2 \\
\hline On va dire + indication de lieu & 1 & - & - & 1 & 0,8 \\
\hline On va dire + indication de temps & 1 & 1 & & 2 & 1,6 \\
\hline Indication de temps + on va dire & 7 & - & - & 7 & 5,6 \\
\hline Adj + On va dire & 11 & - & 1 & 12 & 9,6 \\
\hline Nom + on va dire & 13 & - & 7 & 20 & 16 \\
\hline Verbe + on va dire & 7 & - & 1 & 8 & 6,4 \\
\hline & 96 & 12 & 17 & 125 & 100 \\
\hline
\end{tabular}

Tableau 1 : Distribution de on va dire par structures

On constate que 43,2\% de nos occurrences n'entrent dans aucune des trois catégories suggérées par Lansari (2010), ce qui nous pousse à mettre en cause sa classification. Nous proposerions plutôt une bipartition - correspondant aux modalisations quantative (épistémique) et qualitative 
(dénominationelle) de Lansari - séparant les on va dire portant sur l'énoncé entier et les on va dire portant sur un élément de la phrase. Dans les deux structures, la séquence on va dire peut s'antéposer (généralement suivie d'un que complétif mais pas nécessairement dans la première structure) ou se postposer ${ }^{4}$. Quant à la modalisation qualitative, elle ne se limite pas au nom ou à l'adjectif mais semble pouvoir porter sur toute catégorie lexicale :

(12) [...] on va dire elle a essayé de se suicider, point Ça suffit [...] (CLAPI, les devoirs à la maison)

(13) Elle n'a pas trop de... d'intimité on va dire [...] (DGLFLF, projets-WE_A.)

(14) $[\ldots]$ quand je sors $\mathrm{j}$ 'vais surtout vers euh vers des endroits où $\mathrm{j}$ 'ai : où $\mathrm{j}$ 'ai mes racines on va dire vers rue Saint-Dominique [...] (CFFP2000, 07-02)

Ajoutons que la position finale - que Lansari (2010 : 122) pressentait majoritaire à l'oral - ne représente que $48,8 \%$ de nos occurrences.

\subsubsection{Une structure figée}

Qu'en est-il maintenant du figement de l'emploi modalisant ? Lansari (2010 : 133) considère qu'il « semble éminemment figé » :

- il serait limité à la séquence on va dire ;

- il n'apparaîtrait qu'à la forme affirmative ;

- il ne se réaliserait qu'avec on et ne serait pas possible avec je ;

- il ne serait possible qu'avec un auxiliaire au présent de l'indicatif.

Toutefois, Lansari (2010 : 133-4) relativise ces restrictions : (i) elle donne un exemple où s'insère un adverbe dans la séquence (on va juste dire) et (ii) un exemple négatif (on va pas dire complètement sortie). Pour ce qui est de l'impossibilité d'un je vais dire modalisant, Lansari avait cependant affirmé quelques pages plus haut (p.126) : «Le on de on va dire correspond plutôt, semble-t-il à un je, mais à un je cherchant à s'effacer. »

Un je vais dire modalisant existe bel et bien, comme l'atteste à l'envi le reportage sur un généraliste, devenu célèbre sur internet sous le surnom du «Dr J'vais dire ». Ce médecin, trop fatigué, avait envoyé sa compagne faire une visite à domicile à sa place. Dans le passage cidessous, il rejette l'accusation de faute professionnelle pour les micros du JT de la RTBF le 8 juin 2011 :

(15) Le diagnostic $\boldsymbol{j}$ 'vais dire avait été fait au téléphone donc une dame $\boldsymbol{j}$ 'vais dire enceinte qui se plaint $\boldsymbol{j}$ 'vais dire dans le bas du dos bon ben $\boldsymbol{j}$ 'vais dire euh sur place $\boldsymbol{j}$ 'vais dire j'pouvais rien faire de mieux que de d'entendre et de poser le même acte technique. [...] Comme elle n'est pas $\boldsymbol{j}$ 'vais dire euh du métier $\boldsymbol{j}$ 'vais dire qu'elle n'a pas de diplôme $\boldsymbol{j}$ 'vais dire ça peut $\boldsymbol{j}$ 'vais dire euh étonner plus d'un mais $j$ 'vais dire euh euh moi c'est mon amie et $j$ 'vais dire on s'aime et je sais très bien $j$ 'vais dire qu'elle fait correctement son travail. [...] c'est ça le problème j'vais dire ce qui a troublé la personne c'est qu'on a jeté la seringue et puis qu'on a fait une deuxième qu'on a repris la la seringue de la poubelle mais $j$ 'vais dire ma copine a désinfecté parce qu'elle avait encore plusieurs tampons d'alcool et elle a pris une nouvelle aiguille intra-musculaire donc y a aucun danger $j$ 'vais dire pour la personne.

La contagion gagne aussi la « copine » :

(16) il m'a bien tout expliqué tout c'que j'devais faire comme piquer euh comment prendre euh la seringue $\boldsymbol{j}$ 'vais dire i' m'a tout expliqué. 
La restriction au présent est-elle justifiée? Si nos corpus ne contiennent pas d'occurrences de on allait dire, une recherche avancée sur Google a généré de nombreuses occurrences de cette séquence :

(17) Et une quarantaine de bénévoles se sont mis au service de ce melting-pot des genres avec des grands noms tel qu'Ernesto Tito Puentes, trompettiste "in" (on allait dire dans le vent...) qui dès $21 \mathrm{~h}$, le jeudi 28 , ouvrira la séance sur des rythmes de salsa et de jazz. (http//www.lindependant.fr/2011/07/24/albieres-dix-ans-de-festival-pour-les-musiques-dumonde,44997.php)

(18) Se profilent donc le sapin et les bouboules, les engueulades rituelles et les gavages humains, tout aussi rituels. Aussi allons-nous relayer le très rituel débat du mois de décembre, qui porte, pas tant sur le lâche assassinat de douzaines de milliers d'huitres, mais sur la torture infligée aux canards (et, de moins en moins, aux oies, on allait dire hélas, pour cause de frais de production) afin de produire le foie gras quasiment indétrônable des tables de «fêtes» de fin d'année. (http://www.liberation.f r/vous/0101605926-gavage-le-sondagequi-jette-un-froid-de-canard)

On a l'impression ici que la modalisation n'est plus authentique, mais feinte. L'auteur fait semblant de chercher ses mots mais la reformulation est affectée et déjà rejetée. Elle équivaudrait plutôt à on ne va pas dire :

(17') Et une quarantaine de bénévoles se sont mis au service de ce melting-pot des genres avec des grands noms tel qu'Ernesto Tito Puentes, trompettiste "in" (on ne va pas dire dans le vent...) qui dès $21 \mathrm{~h}$, le jeudi 28 , ouvrira la séance sur des rythmes de salsa et de jazz. (http://www.lindependant.fr/2011/07/24/albieres-dix-ans-de-festival-pour-les-musiques-dumonde,44997.php)

(18') Se profilent donc le sapin et les bouboules, les engueulades rituelles et les gavages humains, tout aussi rituels. Aussi allons-nous relayer le très rituel débat du mois de décembre, qui porte, pas tant sur le lâche assassinat de douzaines de milliers d'huîtres, mais sur la torture infligée aux canards (et, de moins en moins, aux oies, on ne va pas dire hélas, pour cause de frais de production) afin de produire le foie gras quasiment indétrônable des tables de «fêtes» de fin d'année. (http://www.liberation.f r/vous/0101605926-gavage-le-sondage-qui-jette-unfroid-de-canard)

Ce jeu stylistique pourrait être trop sophistiqué dans la production orale spontanée, ce qui expliquerait son absence dans nos corpus.

La séquence $j$ 'allais dire apparaît par contre neuf fois avec un sens modalisant dans le corpus CFPP2000 : sept des occurrences sont produites par un même locuteur :

(19) alors il a fait faillite euh $+j$ 'allais dire euh si j'me trompe pas euh + au début des années soixante-dix il a retrouvé un travail de menuisier + rue d' Paris + justement ++ euh ben juste rue d' Paris y avait une euh une entreprise de menuiserie qui a fermé depuis déjà (CFPP2000, Mo-01)

Ici, on pourrait sans problème remplacer la séquence par on va dire ou je vais dire. On relève les mêmes marqueurs d'hésitation qu'avec on va dire et la modalisation est authentique.

Cette différence de réalisation orale entre on allait dire et $j$ 'allais dire tient au fait qu'avec la première personne, un emploi atténuatif de l'imparfait est possible (je venais vous demander un petit service...). Cette transposition est impossible pour on va dire puisque, par l'utilisation du on, l'émetteur de la séquence sollicite la future coopération de son interlocuteur.

\subsubsection{Un phénomène récent?}


Une dernière hypothèse à vérifier est celle de la récence du phénomène. Lansari (2010) la formule sur base de la quasi-inexistence de l'emploi dans Frantext et sa prédominance à l'oral et dans l'écrit électronique.

Il est évidemment difficile de se prononcer sur la récence du phénomène sans le support d'un corpus diachronique. Dans notre corpus, un élément semble toutefois susceptible de soutenir cette hypothèse : il semble s'agir d'un emploi «jeune». Cet argument est soutenu par la fréquence de la forme dans le corpus DGLFLF composé d'interviews d'étudiants et par la distribution des emplois dans le corpus CFPP2000 où les trois utilisateurs principaux de la séquence sont dans la vingtaine. Par contre, j'allais dire est produit par des gens de plus de 45 ans ( 7 fois par un homme de 58 ans, 1 fois par un autre de 49 ans et 1 fois par une femme de 46 ans) alors qu'une dame de 70 ans produit la majorité (14/31 occurrences) des je dirais.

\subsection{On va dire et ses cousins}

Le verbe dire semble se prêter sous différentes formes à l'expression de la modalisation. Ainsi, Dostie (2004 : 253ss) compte disons au nombre des marqueurs discursifs qu'elle décrit plus en détail. Disons semble partager des contraintes syntaxiques avec on va dire : le marqueur peut s'antéposer avec que ou porter sur un énoncé ou sur un mot :

(20) Disons qu'ici, c'est le salon.

(21) Tu veux aller à ce restaurant-là ? Ben, je le trouve, disons, normal.

Dostie mentionne également d'autres marqueurs dérivés de dire comme je dirai, si je puis dire.... Pour notre part, nous pensons déceler l'émergence d'un je veux dire modalisant dans le corpus CFPP 2000. Il nous faudrait encore approfondir les caractéristiques spécifiques de ces marqueurs...

\subsection{L’interprétation de on va dire dans notre cadre théorique}

Pour rappel, notre projet sur la grammaticalisation des verbes de mouvement aller et venir adopte une approche monosémique : nous postulons que sous la diversité des emplois en discours réside une unité en langue, dérivée de la valeur spatiale originelle. Dans le cas d'aller, cette valeur signifie un mouvement de déplacement dans l'espace extérieur vers un lieu où ne se situe pas (réellement ou fictivement) l'énonciateur principal et / ou l'énonciataire (22) (ce qui, par inférence, peut produire le sens d'éloignement du lieu où se trouve l'énonciateur principal et / ou l'énonciataire (23)).

(22) Demain nous irons au Cimetière marin, à Sète.

Ouais là je pars je vais au labo (conversation).

Ce mouvement est d'orientation ascendante (Guillaume 1929), à savoir qu'il correspond à une représentation selon laquelle le sujet va activement vers le temps.

En tant qu'auxiliaire, aller signifie un mouvement ascendant dans l'espace du dire vers une borne ou un point du procès qui suit, qui représente le lieu où ne se situe pas (réellement ou fictivement) l'énonciateur principal et / ou l'énonciataire.

La périphrase en aller + infinitif convient donc très bien à l'effet modalisant dans lequel l'énonciateur se distancie de son énoncé que ce soit épistémiquement ou dénominationnellement. Cet effet de distanciation est maximisé par la combinaison avec on - 
c'est un moyen terme entre le je et le $t u$ - qui opacifie le point de vue à partir duquel la modalisation s'effectue, et renforce l'idée d'approximation véhiculée par la séquence. La combinaison avec je est également possible quoiqu'impliquant une subjectivité plus grande. Quant à la séquence à la deuxième personne, elle ne réalise pas l'effet modalisant : la distanciation signifiée à l'interlocuteur dans $t u$ vas dire ou vous allez dire implique en retour un rapprochement de l'énonciateur et annule l'effet de modalisation.

\section{Conclusion}

Dans cette étude, nous sommes revenue sur l'emploi dit modalisant de la séquence on va dire à la lueur d'un corpus oral étendu. Sur cette base, nous avons confirmé la double interprétation épistémique ou dénominationnelle de la séquence proposée par Lansari (2010). Par contre, nous avons remis en cause les restrictions paradigmatiques et syntaxiques relevées par cette auteure et étendu l'emploi modalisant aux séquences je vais dire et j'allais dire. Deux pistes de réflexion nous semblent encore devoir être explorées. D'une part, qu'est ce qui démarque les emplois modalisants en aller dire d'autres marqueurs apparentés comme je dirai(s), disons, c'est-à-dire, pour ainsi dire, comment dire... (voir Authier-Revuz 1995)? D'autre part, serait-il possible que l'interprétation dénominationnelle où on va dire est glosable par environ soit plus avancée sur le sentier de la grammaticalisation? Il nous faudra étendre notre corpus avant de pouvoir nous prononcer...

\section{Bibliographie}

Apothéloz, D, (2003). La rection dite «faible» grammaticalisation ou différentiel de grammaticité, Verbum $X X V-3,241-262$.

Authier-Revuz, J. (1995). Ces mots qui ne vont pas de soi. Boucles réflexives et noncoïncidentes du dire. Paris : Larousse.

Branca-Rosoff, S., Fleury, S., Lefeuvre, F. \& Pires, M. (2009). Constitution et exploitation d'un corpus de français parlé parisien, disponible à : http://cfpp2000.univparis3.fr/CFPP2000.pdf

Bres, J. \& Labeau, E. (2012). De la grammaticalisation des formes itive (aller) et ventive (venir) : valeur en langue, emplois en discours, In Rihs, A. \& De Saussure, L. (eds). Etudes de sémantique et pragmatique françaises. Bern : Peter Lang, 143-166.

Bres, J. \& Labeau, E. (à par.). Les auxiliaires aller et venir et l'allure extraordinaire, Journal of French Language Studies.

Dostie, G. (2004). Pragmaticalisation et marqueurs discursifs : Analyse sémantique et traitement lexicographique. Bruxelles : De Boeck / Duculot.

Guillaume, G. (1929). Temps et verbe : théorie des aspects; des modes et des temps. Paris : Honoré Champion.

Lansari, L. (2010). On va dire : vers un emploi modalisant d'aller + infinitif, in Moline, E. \& Vetters, C. (eds.) Temps, aspect et modalité en français (Cahiers Chronos 21). Amsterdam / New York : Rodopi, 119-139.

Schneider, S. (2007) Reduced Parenthetical Clauses as Mitigators : A corpus study of spoken French, Italian and Spanish. Amsterdam : Benjamins, 
${ }^{1}$ Autonyme : élément du langage qui a le pouvoir de référer à son propre signe.

${ }^{2}$ Méta-énonciatif : autoreprésentation du dire en train de se faire (Authier-Revuz $1995: 66$ ).

${ }^{3}$ Dans la Théorie des Opérations Enonciatives, la notion désigne « les représentations mentales associées aux mots avant même qu'il y ait véritablement langage » (Lansari $2010: 128)$.

${ }^{4}$ L'exemple de on va dire en position finale fourni par Lansari : Ca, c'est la version "officielle" on va dire nous semble prouver que la modalisation porte ici sur l'adjectif officielle entre guillemets et produit le même effet que dans la deuxième catégorie de Lansari (on va dire + GN / Adj.). 